ten “ und im Frühjahr 2013 das Treffen des Regionalgruppenbeirats zusammen mit den Augsburger Kolleginnen in Augsburg.
Für uns kann es gerne die nächsten 30 Jahre in München/Südbayern so weitergehen - zum Wohle und Gedeihen des djb und unseren gemeinsamen rechtspolitischen Zielen!

\title{
Landesverband Berlin ehrt zwei langjährige Verbandsmitglieder
}

\author{
Sommerempfang am 12. Juni 2012
}

Zum traditionellen Sommerempfang des djb-Landesverbands Berlin fanden sich am 12. Juni 2011 rund 250 djb-Mitglieder und illustre Gäste aus Politik und Gesellschaft im Pfefferberg im Prenzlauer Berg ein. Im Mittelpunkt des diesjährigen Sommerempfangs standen die Ehrungen von Dr. h.c. Renate Jaeger und Johanna Bacher. Beide erhielten die djb-Ehrennadel für ihre 35-jährige Mitgliedschaft im Deutschen Juristinnenbund.

Die Laudatorinnen Ursula Raue für Johanna Bacher und Dr. Christine Fuchsloch für Dr. h.c. Renate Jaeger gaben in ihren Reden interessante Einblicke in Leben und Wirken der Jubilarinnen. Dabei spannten sie den Bogen vom beruflichen und persönlichen Werdegang der Jubilarinnen zu deren langjähriger Verbundenheit bis hin zum vielfältigen Engagement für den djb.

Der Beifall für die beiden Jubilarinnen hallte noch lange nach und so nahm ein lebhafter Sommerabend seinen Lauf. Der Veranstaltungsort des ehemaligen großräumigen Brauereigeländes, das mittlerweile in höchst unterschiedlicher Weise für Kulturveranstaltungen und Restaurantbetriebe genutzt wird, lud zudem zu einem vorab-Blick auf den in Berlin sehr umstrittenen Standort des temporären Guggenheim Labs ein. Für vielfältigen Gesprächs- und Diskussionsstoff war damit bereits auch ganz ohne Gleichstellungsthemen gesorgt, um den Abend auf der Terrasse des Pfefferbergs bei angeregten Gesprächen ausklingen zu lassen.

\section{Johanna Bacher}

war stellvertretende Direktorin des Amtsgerichts Pankow/Weißensee, ist verheiratet und hat zwei Kinder. Sie wurde 1937 in
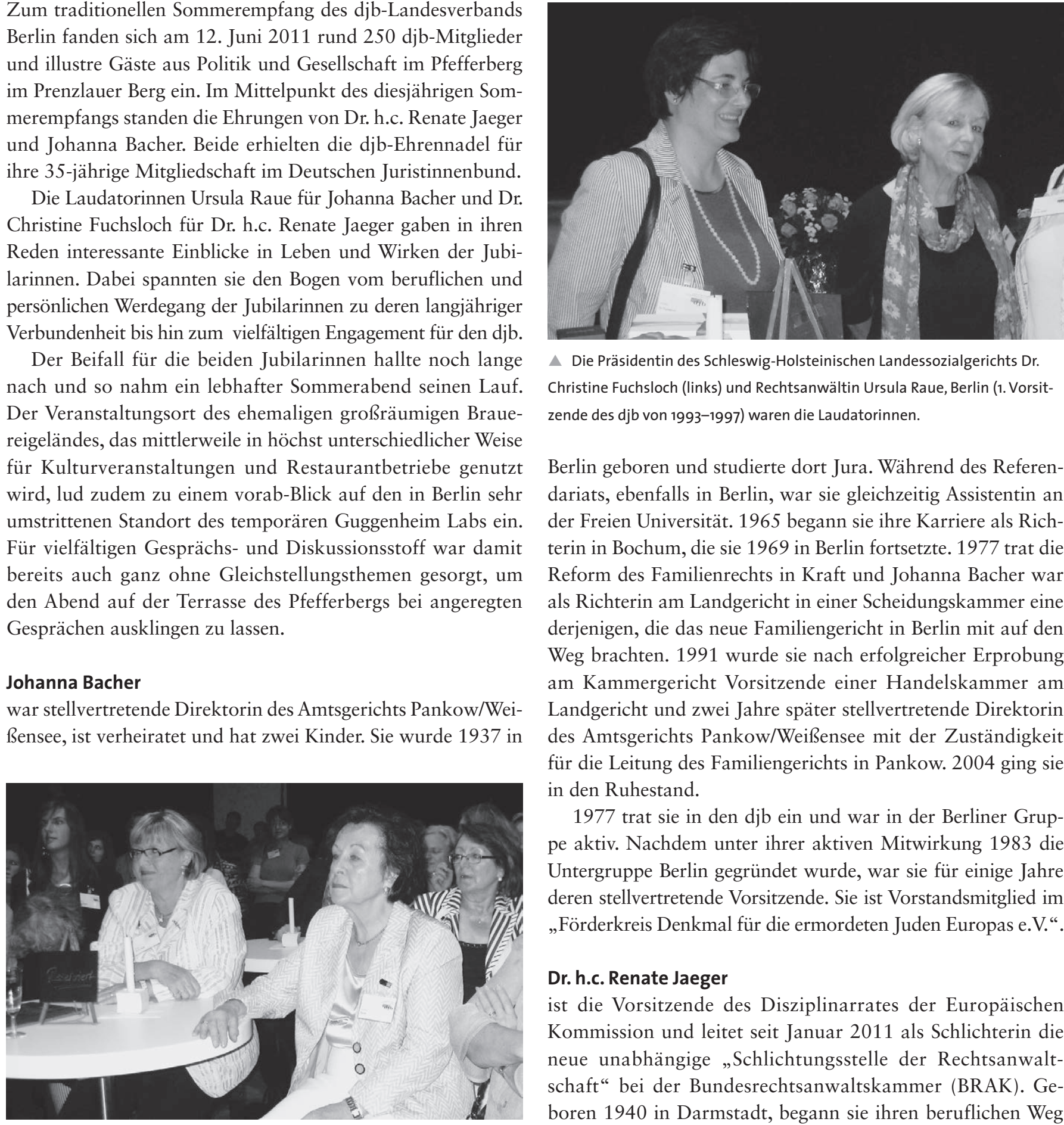

$\Delta$ Die Präsidentin des Schleswig-Holsteinischen Landessozialgerichts Dr. Christine Fuchsloch (links) und Rechtsanwältin Ursula Raue, Berlin (1. Vorsitzende des djb von 1993-1997) waren die Laudatorinnen.

Berlin geboren und studierte dort Jura. Während des Referendariats, ebenfalls in Berlin, war sie gleichzeitig Assistentin an der Freien Universität. 1965 begann sie ihre Karriere als Richterin in Bochum, die sie 1969 in Berlin fortsetzte. 1977 trat die Reform des Familienrechts in Kraft und Johanna Bacher war als Richterin am Landgericht in einer Scheidungskammer eine derjenigen, die das neue Familiengericht in Berlin mit auf den Weg brachten. 1991 wurde sie nach erfolgreicher Erprobung am Kammergericht Vorsitzende einer Handelskammer am Landgericht und zwei Jahre später stellvertretende Direktorin des Amtsgerichts Pankow/Weißensee mit der Zuständigkeit für die Leitung des Familiengerichts in Pankow. 2004 ging sie in den Ruhestand.

1977 trat sie in den djb ein und war in der Berliner Gruppe aktiv. Nachdem unter ihrer aktiven Mitwirkung 1983 die Untergruppe Berlin gegründet wurde, war sie für einige Jahre deren stellvertretende Vorsitzende. Sie ist Vorstandsmitglied im „Förderkreis Denkmal für die ermordeten Juden Europas e.V.“.

\section{Dr. h.c. Renate Jaeger}

ist die Vorsitzende des Disziplinarrates der Europäischen Kommission und leitet seit Januar 2011 als Schlichterin die neue unabhängige „Schlichtungsstelle der Rechtsanwaltschaft" bei der Bundesrechtsanwaltskammer (BRAK). Geboren 1940 in Darmstadt, begann sie ihren beruflichen Weg als Richterin am Sozialgericht Düsseldorf. Nach Stationen 


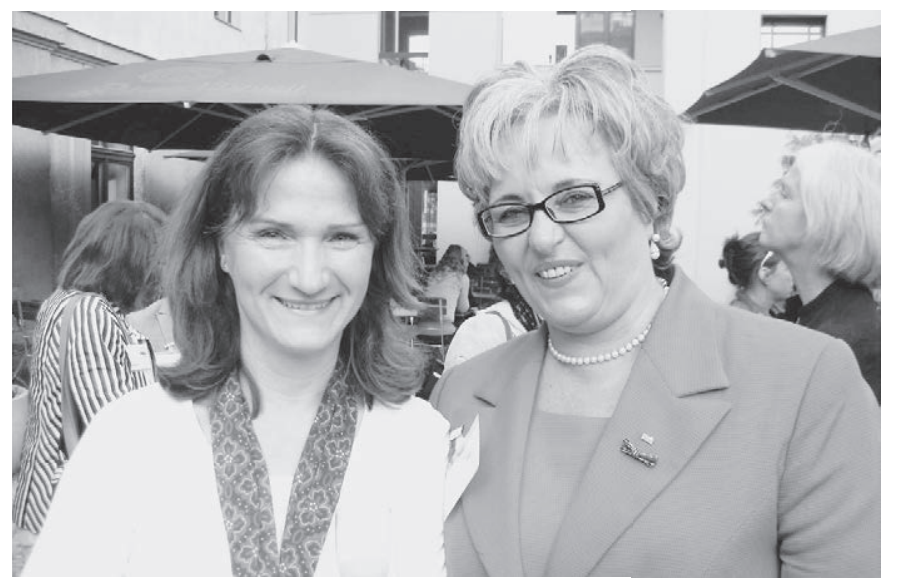

$\Delta$ Rechtsanwältin Prof. (Asoc.) Dr. Jutta Glock (rechts, Vorsitzende des djbLandesverbands Berlin) mit Kathrin Otto (Oberregierungsrätin beim Umweltbundesamt, Stellv. Vorsitzende des djb-Landesverbands Berlin). als wissenschaftliche Mitarbeiterin beim Bundessozial- und Bundesverfassungsgericht, Richterin und Vors. Richterin am Landessozialgericht Nordrhein-Westfalen, Richterin am Bundessozialgericht und Richterin des Bundesverfassungsgerichts wechselte sie am 1. November 2004 als Richterin an den Europäischen Gerichtshof für Menschenrechte (EGMR) in Straßburg.

Seit 1977 djb-Mitglied, war sie jahrelang Mitglied der Rentenrechtskommission und hat zahlreiche Stellungnahmen unseres Verbandes für das Bundesverfassungsgericht erarbeitet sowie diese dort in mündlicher Verhandlung vertreten. Von 1983 bis 1989 war sie Mitglied des Bundesvorstands.

Sie ist Trägerin des Bundesverdienstkreuzes mit Stern und Schulterband sowie Ehrendoktorin der Juristischen Fakultät der Westfälischen Wilhelms-Universität Münster.

\section{Bessere Gesetze aus Brüssel - die Agenda „Intelligente Regulierung" der EU-Kommission}

\section{Sommerempfang der Regionalgruppe Karlsruhe am 21. Juni 2012}

\section{Dr. Anna-Dorothea Polzer, LL.M.}

RG Karlsruhe; Rechtsanwältin, Mannheim

Die Regionalgruppe Karlsruhe des djb konnte zu ihrem diesjährigen Sommerempfang über 100 Gäste begrüßen, darunter Ehrengäste aus Bundesverfassungsgericht und Bundesgerichtshof sowie Gerichts- und Behördenleitungen. Wie auch in den vergangenen Jahren hatte der Bundesgerichtshof seine Pforten - einschließlich derjenigen zum Casino - für die Veranstaltung geöffnet. Den Gästen wurde eine Führung durch alte und neue Gerichtsgebäude geboten, die regen Zuspruch fand.

Das Grußwort sprach die Vizepräsidentin des djb und Bundesanwältin Eva Schübel. Sie forderte erneut eine stärkere Berücksichtigung von Frauen bei der Besetzung von Bundesrichterstellen und die Beseitigung der bestehenden Hemmnisse für

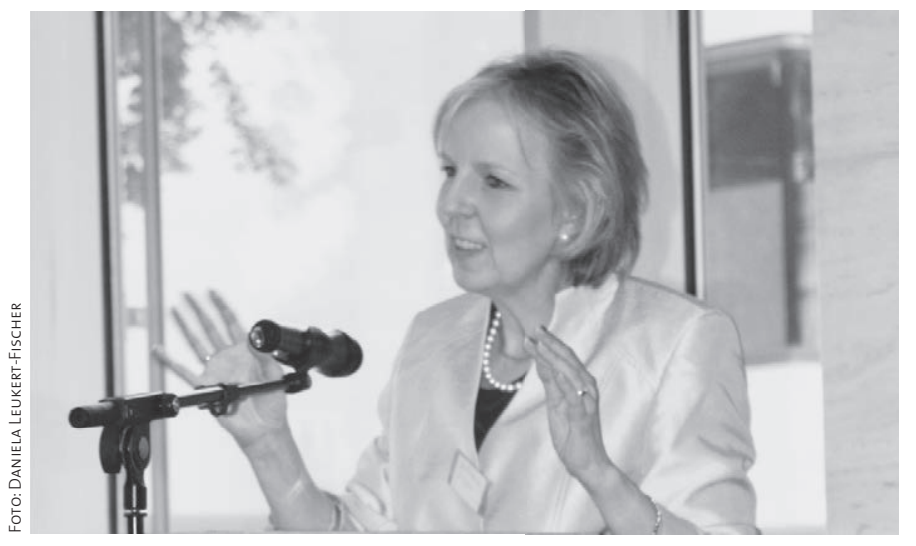

D Dr. Marianne Klingbeil, stellvertretende Generalsekretärin der EU-Kommission. die Karriere von Frauen in der Justiz. Ihr Plädoyer fand große Zustimmung nicht zuletzt bei ihren Kolleginnen in den höheren Positionen der Justiz.

Mittelpunkt des Abends war der Vortrag von Dr. Marianne Klingbeil, stellvertretende Generalsekretärin der EU-Kommission und Mitglied des djb, zum Thema „Transparente Vorbereitung, intelligente Regulierung: Bessere Gesetze aus Brüssel“. Dr. Klingbeil ist promovierte Volkswirtin und leitet im Generalsekretariat den Bereich „Intelligente Gesetzgebung, Evaluation, Folgenabschätzung und Anwendung des EURechts“, der vor allem Querschnittsaufgaben für alle anderen Generaldirektionen wahrnimmt. Die RG Karlsruhe hätte sich keine berufenere Referentin für das Thema des Abends wünschen können.

Die Agenda „bessere“ oder „intelligente Regulierung“ nimmt alle Phasen einer EU-Norm in den Blick: die Vorbereitung durch die Kommission ebenso wie die Annahme durch EU-Parlament und Rat und die Durchführung durch die Mitgliedstaaten. Schließlich ist die Evaluierung und Revision geltenden EU-Rechts durch die Kommission wesentlicher Bestandteil „,intelligenter Regulierung“.

Zur „besseren“ Vorbereitung gehört mittlerweile die frühzeitige Information betroffener Personen oder Unternehmen durch Veröffentlichung lediglich angedachter Gesetzgebungsvorhaben, sogenannter „roadmaps“, ein bis zwei Jahre vor deren Annahme, die einerseits ein rechtzeitiges Reagieren, aber auch längerfristige Vorbereitung auf die aktive Teilnahme an den Konsultationen zum eigentlichen Regulierungsentwurf ermöglicht. Die Referentin lud die Teilnehmer/innen ein, sich selbst - wie es jede/r andere Interessierte auch kann - im In- 